\title{
Comparación in vitro de la profundidad de curado de una resina nanohíbrida fotoactivada con luz halógena versus luz led
}

\author{
In Vitro Comparison of the Curing Depth of a Nanohybrid \\ Resin Photoactivated by Halogen Light Versus LED Light
}

Ana Gabriela Guerrero Bravo, Od. ${ }^{1}$, Raúl Edison Chumi Terán $\bowtie$, MSc. $^{2}$

\author{
1 Práctica privada \\ ${ }^{2}$ Facultad de Odontología, Universidad de Educación, Ecuador
}

Universidad de Educación, Cuenca, Ecuador, Mariano Cueva 856. Correo electrónico: raulchumit@hotmail.com

Recibido: 26 de abril del 2017 Aprobado: 1 de junio del 2017

Cómo citar este artículo: Guerrero-Bravo AG, Chumi-Terán R. Comparación in vitro de la profundidad de curado de una resina nanohíbrida fotoactivada con luz halógena versus luz led. Rev Nac Odontol. 2018;13(26):1-10. doi: http://dx.doi. org/10.16925/od.v13i26.2042

\section{Resumen}

Introducción: comparar la profundidad de curado de una resina nanohíbrida fotoactivada con luz halógena versus luz led según iso 4049.

Materiales y métodos: metodología transversal actual, descriptiva y comparativa de laboratorio. Se utilizó un formador de probetas. Se puso en la base del formador de probetas una tira de celuloide, sobre la cual se puso la resina z350 xt (3M), color A2, esmalte. Se sobreobturó la altura del formador de probetas y se puso una segunda tira de celuloide en la parte superior. Se fotoactivó la resina con una lámpara halógena convencional y una lámpara led de tercera generación. Se removió el espécimen del formador y se eliminó el material en estado plástico sin polimerizar con una espátula. Se midió la altura del material curado con el micrómetro digital (Т304B.w-1220) a una exactitud de $+0,01 \mathrm{~mm}$. Esta prueba se realizó tres veces y se obtuvo el valor promedio de la profundidad de curado. Al dividirlo por dos se obtuvo la profundidad de curado según Iso 4049. Se aplicaron pruebas estadísticas de normalidad (Kolmogorov-Smirnov(a) y Shapiro-Wilk) y pruebas no paramétricas (Mann-Whitney) para comprobar si los valores siguen una distribución normal. Se aplicó la prueba t de Student para determinar si hay una diferencia estadísticamente significativa entre los resultados.

Resultados: el valor mínimo de profundidad de curado se obtuvo con la lámpara halógena (2,23 mm), a diferencia de la fotoactivación con la lámpara led (2,32 mm).

Conclusión: existen diferencias significativas en la profundidad de curado de una resina cuando es fotopolimerizada con luz halógena versus luz led.

Palabras clave: fotoactivación, Iso 4049, profundidad de curado, resinas nanohíbridas. 


\title{
In Vitro Comparison of the Curing Depth of a Nanohybrid Resin Photoactivated by Halogen Light Versus LED Light
}

\begin{abstract}
Aim: To compare the curing depth of a nanohybrid resin photoactivated by halogen light versus LED light according to iso 4049.

Materials and methods: Current cross-sectional, descriptive, comparative laboratory method. A specimen former was used. A strip of celluloid was placed on the base of the specimen former, on which the resin z350 xt (3M), shade A2 and enamel was put. The height of the specimen former was overfilled, and a second strip of celluloid was placed on top. The resin was photoactivated by a conventional halogen lamp and a third-generation LED lamp. The specimen was removed from the former and the material in a plastic state without polymerization was removed with a spatula. The height of the cured material was measured with the digital micrometer (T304B.w-1220) at an accuracy of $+0.01 \mathrm{~mm}$. This test was performed three times and the average value of the curing depth was obtained. When dividing it by two, the curing depth was obtained according to iso 4049. Statistical normality tests (Kolmogorov-Smirnov(a) and Shapiro-Wilk) and nonparametric tests (Mann-Whitney) were performed to check if the values follow a normal distribution. The Student's t test was used to determine if there is a statistically significant difference between the results.
\end{abstract}

Results: The minimum curing depth value was obtained with the halogen lamp $(2.23 \mathrm{~mm})$, unlike photoactivation by LED lamp $(2.32 \mathrm{~mm})$.

Conclusion: There are significant differences in the curing depth of a resin when photopolymerized by halogen light versus LED light.

Keywords: photoactivation, Iso 4049, curing depth, nanohybrid resins.

\section{Comparação in vitro da profundidade de cura de uma resina nano-híbrida fotoativada com luz halógena versus luz LED}

Resumo

Introdução: comparar a profundidade de cura de uma resina nano-híbrida fotoativada com luz halógena versus luz LED segundo iso 4049.

Materiais e métodos: metodologia transversal atual, descritiva e comparativa de laboratório. Utilizou-se um formador de provetas. Pôs-se na base do formador de provetas uma fita de celuloide, sobre a qual se pôs a resina z350 xt (3M), cor A2, esmalte. Sobreobturou-se a altura do formador de provetas e pôs-se uma segunda fita de celuloide na parte superior. Fotoativou-se a resina com uma lâmpada halógena convencional e uma lâmpada LED de terceira geração. Removeu-se a amostra do formador e eliminou-se o material em estado plástico sem polimerizar com uma espátula. Mediu-se a altura do material curado com o micrômetro digital (T304B.w-1220) a uma exatidão de $+0,01 \mathrm{~mm}$. Esse teste foi realizado três vezes e foi obtido o valor médio da profundidade de cura. Ao dividi-lo por dois, obteve-se a profundidade de cura segundo iso 4049. Aplicaram-se estatísticas de normalidade (Kolmogorov-Smirnov(a) e Shapiro-Wilk) e testes não paramétricos (Mann-Whitney) para comprovar se os valores continuam uma distribuição normal. Aplicou-se o t de Student para determinar se há uma diferença estatisticamente significativa entre os resultados.

Resultados: o valor mínimo de profundidade de cura foi obtido com a lâmpada halógena (2,23 mm), à diferença da fotoativação com a lâmpada LED (2,32 mm).

Conclusão: existem diferenças significativas na profundidade de cura de uma resina quando é fotopolimerizada com luz halógena versus luz LED.

Palavras-chave: fotoativação, Iso 4049, profundidade de cura, resinas nano-híbridas. 


\section{Introducción}

Hoy en día, los materiales más utilizados para la ejecución de técnicas hechas a mano en una sola cita son los composites, que son los agentes protagónicos para técnicas directas. Según autores como Rodríguez y Pereira [1], "sus grandes posibilidades estéticas le dan variadas indicaciones terapéuticas, que se incrementan gracias a la gran versatilidad de presentaciones que ofrecen". Sin embargo, debido a que la adhesión de este tipo de materiales no depende de cómo realicemos la cavidad sino de la técnica adhesiva, hay una mayor posibilidad de preservar y mantener intacta una mayor cantidad de estructura dental. Para lograr el éxito en la clínica durante un tratamiento con estos materiales, debemos tomar en cuenta ciertos aspectos trascendentales como: buen aislamiento (de preferencia absoluto), material e instrumental restaurador adecuado, protocolos apropiados para técnica adhesiva dental y manipulación correcta de los tiempos de polimerización.

Con el pasar del tiempo, los composites han evolucionado mejorando totalmente sus cualidades y sus propiedades físicas, químicas, mecánicas, ópticas, estéticas y funcionales; las resinas que se han perfeccionado y desarrollado notablemente en el campo adhesivo en la última década son las resinas o composites nanohíbridas, las cuales "están constituidas por partículas con tamaños menores a $10 \mathrm{~nm}$, mismas que se disponen de una manera individual o conforman dos grupos (nanoclusters o nanoagregados) con un tamaño no superior a 75 $\mathrm{nm}$ " [1]. "Este tipo de resinas con nanotecnología ofrecen alta translucidez, pulido superior, disminución notable de la microfiltración y mayor resistencia al desgaste" [2].

Las resinas nanohíbridas, al estar constituidas por micropartículas, van a generar una contracción menor en el momento de la polimerización y por lo tanto generan menor estrés de contracción en la restauración, menor flexión cuspídea y sobre todo disminución notable tanto macro como microscópica de las fisuras que se forman entre la interfase diente-restauración a nivel de los bordes adamantinos, garantizando así la vida útil de las restauraciones.

En 1963, R. L. Bowen desarrolló un composite con características estéticas similares a los dientes naturales, formando el eldimetacrilato de diglicidilbisfenol A (BIS-GMA), la base o el núcleo de las actuales resinas compuestas.

Esta molécula BIS-GMA fue el resultado de diversos ensayos clínicos a base de resina epóxica, acrílica y partículas de relleno de refuerzo. La partícula con sílice es recubierta por un agente de enlace, "el vinil silano, para lograr la unión química entre ella y el BIS-GMA: por su grupo silano se une a la partícula y por su grupo vinilo, al BIS-GMA" [3].

Según el tamaño de las partículas, las resinas compuestas se clasifican en:

- Macropartículas

- Micropartículas

- Híbridas

- Microhíbridas

- Nanopartículas

Los componentes de las resinas compuestas son:

- Matriz orgánica

- Agentes iniciadores

- Relleno inorgánico

- Agente de enlace

- Otros componentes (pigmentos, aditivos, inhibidores)

Ambas fases, inorgánica y orgánica, están unidas química y micromecánicamente [4] (Tabla 1).

Tabla 1. Composición de las resinas y función de los componentes

\begin{tabular}{cc}
\hline Componente & Función \\
\hline BIS-GMA, UDMA & Matriz resinosa \\
\hline $\begin{array}{c}\text { Cuarzo, cristal de Ba, sr, y zr, } \\
\text { silicato de tamaño coloidal, } \\
\text { silicato de zn. }\end{array}$ & Partículas de carga \\
\hline $\begin{array}{c}\text { Titanatos, zirconatos, } \\
\text { organosilanos }\end{array}$ & Agentes de unión o silanos \\
\hline Amina terciaria & Iniciador \\
\hline Canforquinona & Fotoiniciador \\
\hline Hidroquinona, benzoquinona & Inhibidor \\
\hline Óxido de aluminio, dióxido & Modificadores ópticos \\
\hline
\end{tabular}

Fuente: [5] 


\section{Otros componentes}

- Pigmentos o colorantes.

- Aditivos, absorben la luz uv y dan estabilidad al color.

- Inhibidores, evitan que exista polimerización prematura del material; los más usados son benzoquinona, hidroquinona y derivados del fenol.

"La polimerización es un proceso químico por el cual los monómeros de la matriz de resina se agrupan entre sí dando lugar a una molécula de gran peso, llamada polímero, que puede ser una molécula lineal o tridimensional" [4]. Dicho concepto no se debe confundir con la profundidad de curado, que según Krämer [6] es definida como "la disminución de la intensidad de la luz desde la superficie hacia la profundidad del composite, causado por la absorción y dispersión de la luz". Como ejemplo, el 50\% de la energía lumínica que llega al composite se pierde a solo $0,5 \mathrm{~mm}$ de profundidad, a $1 \mathrm{~mm}$ llega el $25 \%$, a $2 \mathrm{~mm}$ llega el $9 \%$ y a $3 \mathrm{~mm}$ llega el 3\% [6]. Esta disminución de la intensidad resulta en lo que comúnmente llamamos profundidad de curado.

También, definen la profundidad de curado como "el nivel en que el valor de dureza es equivalente a por lo menos $90 \%$ de la dureza en la parte superior del composite" [6]. La porción más externa del composite que se encuentra más cerca a la lámpara de fotocurado tendrá una polimerización más completa, debido a que presenta una mayor cantidad de fotoiniciadores y radicales libres que se convertirán después en una cadena de polímeros. La dureza del composite en la porción más externa asegura la fotopolimerización pero no una correcta profundidad de curado.

\section{Factores que afectan la profundidad de curado}

- El tipo de resina compuesta (macromecánica, micromecánica, nanohíbrida, etc.).

- Los incrementos de resina, que no deben ser mayores a $2 \mathrm{~mm}$ de espesor.

- La distancia de la luz medida desde la punta de la lámpara a la superficie de la resina.

- El tiempo y la potencia de radiación.

- El tamaño y la distribución de las partículas de carga [7].

\section{Unidades de fotopolimerización}

Las lámparas de fotopolimerización han evolucionado sustancialmente en un corto periodo de tiempo, desarrollando nuevas tecnologías de producción y de uso [8]. Se clasifican según el origen del haz radiante:

- Convencional o lámpara halógena

- Arco de plasma

- Láser de argón

- Led [8]

Las lámparas, en general, deben activar los fotoiniciadores encontrados en los materiales resinosos para poder producir la fotopolimerización [8]. Entre los fotoiniciadores se pueden mencionar: "la canforquinona, cuyo rango de activación es de 400 a $500 \mathrm{~nm}$; el 1 fenil- 1,2 propandiona activable entre los 360 y $480 \mathrm{~nm}$; leucerina activable entre los 350 y $430 \mathrm{~nm}$ " [9]. Las lámparas de fotocurado que utilicemos deben de estar acordes al rango de longitud de onda que requiera cada fotoiniciador.

\section{Norma Iso 4049}

La Organización Internacional de Normalización (Iso, por sus siglas en inglés), con el objetivo de garantizar una correcta profundidad de curado de las resinas compuestas durante la ejecución de las restauraciones directas, determinó un método para definir el grosor máximo de los incrementos de resina compuesta; este método es oficialmente denominado como "Iso 4049 actualización 2011" [10]. Esta norma internacional especifica los requisitos para los materiales restauradores a base de resina para el uso primario en restauraciones directas.

La Iso desarrolló una especificación técnica para la medición de la intensidad de la luz de curado. Así, sugiere una mínima intensidad de $300 \mathrm{mw} / \mathrm{cm}^{2}$ entre una amplitud de banda de 400 a $515 \mathrm{~nm}$ de la luz de curado. Además, para materiales de relleno fotopolimerizables, requiere que los composites tengan un mínimo de profundidad de curado de $1,5 \mathrm{~mm}$ cuando son irradiados con el tiempo recomendado por el fabricante [11].

En algunas ocasiones, el profesional de la salud dental no sigue el protocolo adecuado para el fotocurado de resinas, el cual indica que no debe haber una distancia de separación mayor a $1 \mathrm{~mm}$ entre 
la lámpara de fotocurado y el material de restauración, ya que la intensidad de la luz de la lámpara disminuye mientras más se aleje del composite. "Si la distancia desde la lámpara a la superficie del composite es mayor a $3 \mathrm{~mm}$, la intensidad lumínica se reduce al $40 \%$ e inclusive pudiendo llegar a un $23 \%$ de reducción lumínica afectando la profundidad de curado" [12].

Para que la resina compuesta evaluada apruebe la Norma Iso 4049 en lo que a profundidad de curado se refiere, esta prueba debe realizarse tres veces, y en el total de estas pruebas la profundidad de curado no debe ser menor a $2 \mathrm{~mm}$ ni mayor a $0,5 \mathrm{~mm}$ por debajo del valor establecido por el fabricante.

Este tema de investigación es un punto de partida para futuras investigaciones en el Ecuador, ya que es un tema poco explorado según los parámetros y criterios que fueron usados en este trabajo (Norma Iso 4049). Este método es una forma sencilla y confiable de medir la profundidad de curado en relación con otros métodos como los perfiles de dureza Vickers.

Por las razones señaladas, se planteó la hipótesis de que existen diferencias significativas en la profundidad de curado de las resinas nanohíbridas fotoactivadas con luz led y luz halógena convencional. El objetivo general fue: comparar in vitro la profundidad de curado de la resina nanohíbrida fotoactivada con luz halógena versus luz led según la Norma Iso 4049. Los objetivos específicos de este trabajo fueron:

- Medir la profundidad de curado de las muestras de la resina z350 xt, A2, esmalte, al aplicar luz halógena según la Norma Iso 4049.

- Medir la profundidad de curado de las muestras de la resina z350 xt, A2, esmalte, al aplicar luz led según la Norma iso 4049.

Las variables de la investigación fueron:

- Fuente de luz (lámpara halógena, lámpara led)

- Profundidad de curado (micrómetro manual calibrado a $+0,01 \mathrm{~mm}$ )

\section{Materiales y métodos}

Para evaluar la profundidad de curado de una resina, la iso exige ciertos materiales para la elaboración de las muestras como son:
- Formador de probetas para la preparación de una muestra cilíndrica de $6 \mathrm{~mm}$ de largo y $4 \mathrm{~mm}$ de diámetro, a menos que el fabricante indique una profundidad de curado con exceso de $5 \mathrm{~mm}$; en tal caso, el molde debe ser de al menos $2 \mathrm{~mm}$ más largo que el indicado para la profundidad de curado requerida.

- Dos losetas o portaobjetos, cada uno con suficiente área para cubrir la orilla u orificio del formador de probetas.

- Banda matriz de celuloide $(50+30) \mathrm{mm}$ de grosor.

- Energía de origen externo, es recomendada por el fabricante para usarse en la prueba de este material. En este caso, se utilizó una lámpara de fotocurado led modelo SDI de tercera generación marca Radii Plus y una lámpara halógena convencional Qhl75 marca Dentsply".

- Micrómetro digital, con exactitud de $+0,01 \mathrm{~mm}$ (т304B.w-1220).

- Espátula de plástico.

- En este trabajo, se utilizó composite z350 xt de la marca comercial $3 \mathrm{M}^{\circ}, \mathrm{A} 2$, esmalte.

Este estudio corresponde a una investigación de laboratorio in vitro, en el cual se estableció en 46 el número de muestras de composite z350 xt de la marca comercial $3 \mathrm{M}^{\circ}$, color $\mathrm{A} 2$, esmalte, tanto para la fotoactivación con luz halógena como 46 muestras para la fotoactivación con luz led.

Estas resinas fueron polimerizadas según las indicaciones de la casa comercial, utilizando una lámpara de luz halógena convencional y una lámpara de luz led de tercera generación modelo sDI. Para la toma de las muestras, se siguió el procedimiento que se muestra en la Tabla 2.

Se hizo un promedio de la intensidad emitida por la guía de la luz de lámpara halógena, y con un radiómetro se tomaron 10 muestras y nos dio un promedio de $435 \mathrm{mw} / \mathrm{cm}^{2}$.

Se hizo un promedio de la intensidad emitida por la guía de la luz de lámpara led, y con un radiómetro se tomaron 10 muestras y nos dio un promedio de 1195,5 mw/ $\mathrm{cm}^{2}$ (Tabla 3).

Después, se puso en la base del formador de probetas una tira de celuloide, sobre la cual se puso la resina siguiendo las especificaciones del fabricante, teniendo en cuenta eliminar las burbujas de aire. Ligeramente, sobreobturamos el formador de probetas y se puso una segunda tira de celuloide en la parte superior. Se presionó el formador 
Tabla 2. Lámpara halógena Dentsply

\begin{tabular}{cccccccccccc}
\hline N. disparo & $\mathbf{1}$ & $\mathbf{2}$ & $\mathbf{3}$ & $\mathbf{4}$ & $\mathbf{5}$ & $\mathbf{6}$ & $\mathbf{7}$ & $\mathbf{8}$ & $\mathbf{9}$ & $\mathbf{1 0}$ & Promedio \\
\hline $\mathrm{MW} / \mathrm{cm}^{2}($ Low $)$ & 1195 & 1195 & 1190 & 1190 & 1200 & 1195 & 1200 & 1195 & 1195 & 1200 & 1195,5 \\
\hline
\end{tabular}

Fuente: elaboración propia

Tabla 3. Lámpara led SDI

\begin{tabular}{cccccccccccc}
\hline N. disparo & $\mathbf{1}$ & $\mathbf{2}$ & $\mathbf{3}$ & $\mathbf{4}$ & $\mathbf{5}$ & $\mathbf{6}$ & $\mathbf{7}$ & $\mathbf{8}$ & $\mathbf{9}$ & $\mathbf{1 0}$ & Promedio \\
\hline $\mathrm{MW} / \mathrm{cm}^{2}(\mathrm{Low})$ & 1195 & 1195 & 1190 & 1190 & 1200 & 1195 & 1200 & 1195 & 1195 & 1200 & 1195,5 \\
\hline
\end{tabular}

Fuente: elaboración propia

de probetas y la tira de celuloide sobre la loseta para eliminar el exceso de material. Rueggeberg [13] indica que con una unidad de luz halógena el tiempo recomendado para la fotopolimerización es de cuarenta segundos; por lo tanto, es el tiempo que se irradiaron las muestras de composites sin distancia de la guía de la luz, y con la lámpara led de tercera generación se irradió 10 segundos siguiendo las instrucciones del fabricante (10 segundos para intensidad de luz $\geq 1200 \mathrm{mw} / \mathrm{cm}^{2}$ ).

Removimos el espécimen del formador de probetas después de completar la exposición de luz, y se eliminó suavemente el material en estado plástico sin polimerizar con una espátula. Medimos la altura del material curado con el micrómetro digital a una exactitud de $+0,01 \mathrm{~mm}$. Registramos este valor $\mathrm{y}$ al dividirlo por dos nos dio la profundidad de curado según la Norma iso 4049.

Para el análisis de datos, utilizamos "la prueba $\mathrm{t}$ en donde se compararon las medias y las desviaciones estándar de cada grupo de datos y se determinó si entre esos parámetros las diferencias son estadísticamente significativas o si solo son diferencias aleatorias" [14].

Este proyecto no implicó ningún conflicto bioético, ya que no afectó la integridad de ningún ser humano, por tanto, los datos recolectados fueron tomados en el laboratorio de biomateriales de la Universidad Católica de Cuenca, Ecuador, Facultad de Odontología.

\section{Resultados}

Interpretación 1: se establecieron 46 muestras para que fueran fotopolimerizadas con la lámpara halógena por 40 segundos a $0 \mathrm{~mm}$ de distancia; y
46 muestras para fotopolimerizarlas con la lámpara led a una distancia de $0 \mathrm{~mm}$ y con un tiempo de exposición de 10 segundos (Tabla 4).

Tabla 4. Distribución de las muestras de resina z350 xt, A2, esmalte, de acuerdo con los grupos de trabajo

\begin{tabular}{ccc}
\hline & Muestra & Porcentaje \\
\hline Halógena & 46 & $50 \%$ \\
Led & 46 & $50 \%$ \\
\hline Total & 92 & $100 \%$ \\
\hline
\end{tabular}

Fuente: elaboración propia

Interpretación 2: la media de la profundidad de curado de la lámpara halógena con un intervalo de confianza del $95 \%$ es de $2,26 \mathrm{~mm}$, con una desviación estándar de $0,02 \mathrm{~mm}$. El valor máximo que se alcanzó fue de 2,29 mm y el valor mínimo fue de 2,23 mm (Tabla 5).

Tabla 5. Estadísticos descriptivos de la profundidad de curado de la lámpara de luz halógena

\begin{tabular}{cc}
\hline Estadísticos & L. halógena \\
\hline Media & 2,26 \\
Mediana & 2,26 \\
Moda & 2,26 \\
Desviación estándar & 0,02 \\
Varianza & 0,00 \\
Coef. variación & 0,01 \\
X mín. & 2,23 \\
X máx. & 2,29 \\
\hline
\end{tabular}

Fuente: elaboración propia 
Interpretación 3: la media de la profundidad de curado de la lámpara led con un intervalo de confianza del $95 \%$ es de $2,29 \mathrm{~mm}$, con una desviación estándar de $0,02 \mathrm{~mm}$. El valor máximo que se alcanzó fue de $2,32 \mathrm{~mm}$ y el valor mínimo fue de 2,25 mm (Tabla 6).

Tabla 6. Estadísticos descriptivos de la profundidad de curado de la lámpara de luz led

\begin{tabular}{cc}
\hline Estadísticos & L. led \\
\hline Media & 2,29 \\
Mediana & 2,29 \\
Moda & 2,29 \\
Desviación estándar & 0,02 \\
Varianza & 0,00 \\
Coef. variación & 0,01 \\
x mín. & 2,25 \\
x máx. & 2,32 \\
\hline
\end{tabular}

Fuente: elaboración propia

Los datos no siguen una distribución normal. Para comparar los grupos, se harán pruebas no paramétricas (Tabla 8 ).

Hay diferencia en la profundidad de curado en una resina al ser fotoactivada con luz halógena versus luz led.

Interpretación 4: al aplicar la t de Student, se pudo constatar que el valor de $\mathrm{p}<0,05$ (Tabla 9). Por lo tanto, la hipótesis sí se aprueba porque sí existe una diferencia significativa en la profundidad de curado cuando los bloques de resina son fotopolimerizados con la lámpara halógena y con la lámpara led.

Tabla 7. Pruebas de normalidad

\begin{tabular}{|c|c|c|c|c|c|c|c|}
\hline & & \multicolumn{3}{|c|}{ Kolmogorov-Smirnov(a) } & \multicolumn{3}{|c|}{ Shapiro-Wilk } \\
\hline \multicolumn{2}{|c|}{ Profundidad de curado } & Estadístico & gl & Sig. & Estadístico & $\mathrm{gl}$ & Sig. \\
\hline Lámpara halógena & $0 \mathrm{~mm}$ distancia &, 373 & 90 &, 000 & 643 & 90 &, 000 \\
\hline Lámpara led & $0 \mathrm{~mm}$ distancia & ,391 & 90 &, 000 & ,626 & 90 &, 000 \\
\hline
\end{tabular}

Tabla 8. Prueba no paramétrica

\begin{tabular}{cc}
\hline Fuente de luz \\
$(\mathbf{0}$ mm distancia $)$ & $\begin{array}{c}\text { Mann-Whitney } \\
\text { p-valor }\end{array}$ \\
\hline Lámpara led & 0,681 \\
Lámpara halógena & 0,000 \\
\hline & 0,000 \\
\hline
\end{tabular}

Fuente: elaboración propia

Tabla 9. Prueba t de Student y comparación de los resultados obtenidos con la luz halógena y con la luz led

\begin{tabular}{ccc}
\hline Estadísticos & L. halógena & L. led \\
\hline Media & 2,26 & 2,29 \\
Mediana & 2,26 & 2,29 \\
Moda & 2,26 & 2,29 \\
Desviación es- & 0,02 & 0,02 \\
tándar & 0,00 & 0,00 \\
Varianza & 0,01 & 0,01 \\
Coef. variación & 0,00000001 & \\
Valor t de Student &
\end{tabular}

Fuente: elaboración propia

Interpretación 5: el valor mínimo de profundidad de curado se obtuvo con la lámpara halógena siendo este valor de $2,23 \mathrm{~mm}$ y su máximo siendo de 2,29 mm; a diferencia de la fotoactivación con la lámpara led, cuya profundidad de curado alcanzó un máximo de 2,32 mm y un mínimo de 2,25 mm (Figura 1).

Fuente: elaboración propia 


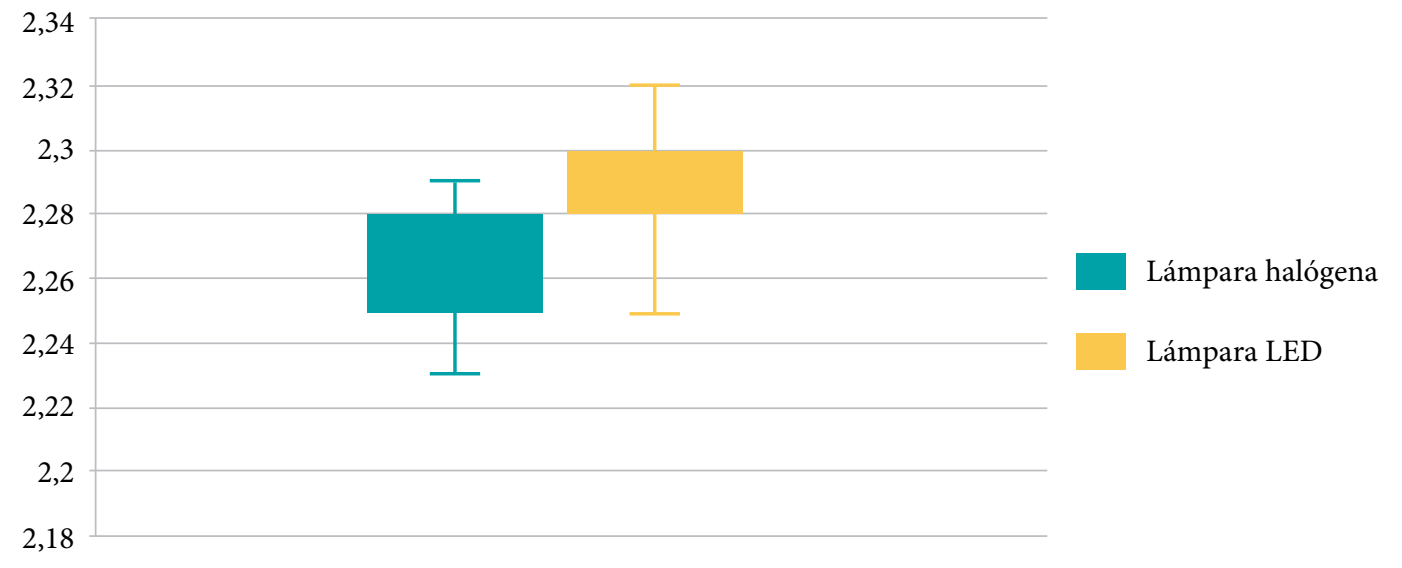

Figura 1. Distribución de la profundidad de curado Fuente: elaboración propia

\section{Discusión}

La profundidad de curado es una medida de la eficiencia de la polimerización, pues el material que quede sin polimerizar puede migrar al medio bucal y producir reacciones alérgicas en algunos pacientes, así como estimular el crecimiento de bacterias alrededor de la restauración [15]. La Norma Iso 4049 exige que estos materiales deben tener profundidad de curado mayor a $2 \mathrm{~mm}$ y la pérdida del material sin polimerizar debe ser inferior a $0,5 \mathrm{~mm}[16]$.

Posterior a todas las pruebas estadísticas realizadas, se pudo constatar que existe un mejor comportamiento de la resina entre los grupos de estudio en cuanto a profundidad de curado cuando se fotopolimeriza con una lámpara led de alta potencia, ya que minimiza el tiempo de exposición de la radiación a una distancia de $0 \mathrm{~mm}$.

Los resultados de esta investigación determinaron que se obtiene una mayor profundidad de curado en una resina nanohíbrida al ser activada con una unidad led de tercera generación que con una lámpara halógena. Estos resultados son similares a los obtenidos en un estudio realizado por Caro et al., en el que concluyó "que es posible lograr una mayor profundidad de polimerización en resinas compuestas mediante la unidad led respecto a la unidad halógena convencional y que esta diferencia es estadísticamente significativa $(\mathrm{p}<0,05)$ " [16].
Se constató que se puede lograr una polimerización correcta en cuerpos de resina compuesta de hasta $2 \mathrm{~mm}$ de grosor con una lámpara convencional y con una lámpara led de tercera generación, dado que, a mayor grosor de la resina, mayor deberá ser la intensidad de la luz y el tiempo de exposición; estos resultados coinciden con los obtenidos en el estudio realizado en el 2014 por Yap y Soh [8]. Aunque ningún autor recomienda que los incrementos de resina superen los $2 \mathrm{~mm}$ de grosor, pues ninguna lámpara de fotoactivación tendría las características para polimerizar incrementos mayores.

En los análisis estadísticos de este proyecto, se observó que hay una diferencia significativa $(\mathrm{p}<0,05)$ entre los dos grupos de muestra al aplicar la t de Student. Estos resultados son similares a los obtenidos en una investigación realizada por Varanes et al. [17], en la cual la mayor profundidad de polimerización la obtuvo con una lámpara de alta potencia (led) a $0 \mathrm{~mm}$ de distancia que con una distancia mayor.

El valor más alto de profundidad de curado fue conseguido con la lámpara led, siendo este valor de $2,32 \mathrm{~mm}$, y el valor más bajo fue de $2,23 \mathrm{~mm}$, conseguido con una lámpara halógena. Estos resultados son similares a los obtenidos por Varanes et al. [17], siendo de 2,39 $\mathrm{mm}$ la profundidad de curado más alta obtenida con una unidad de alta potencia por 10 segundos. 
En el 2005, Varanes et al. [17] realizaron un estudio que "evaluó la profundidad de polimerización de composites dentales fotopolimerizables, entre estas resinas se encontraba la resina Tetric N Ceram; la determinación de la profundidad de polimerización se realizó según la Norma Iso 4049; en los resultados se observó que la profundidad de curado fue de 2,49 $\pm 03 \mathrm{~mm}$ ". Estos resultados tienen un valor un poco mayor a los obtenidos en esta investigación, en la cual la profundidad de polimerización fue de 2,32 $\mathrm{mm}$, valor obtenido bajo los mismos criterios del Iso.

El tiempo de exposición de las muestras de resina se estableció de acuerdo con las indicaciones del fabricante. En el caso de la resina z350 xt, se aplicó por 40 segundos la luz halógena y por 10 segundos la luz de alta potencia. Sin embargo, en un estudio realizado por Shortal [18] se determinó que el tiempo de exposición debe ser mayor al sugerido por el fabricante si se quiere lograr una profundidad de curado adecuada, ya que las condiciones del ambiente pueden variar y por lo tanto afectar la resina.

Este proyecto investigativo fue realizado siguiendo los parámetros establecidos por la Norma Iso 4049, dado que este organismo especifica los requisitos para los materiales restauradores a base de resina, semejante al estudio realizado por Caro [16] según estos mismos parámetros.

\section{Conclusiones}

Existen diferencias significativas en la profundidad de polimerización de cuerpos de resina fotoactivados con luz led en relación con los fotopolimerizados con luz halógena. El valor obtenido de la profundidad de curado de las muestras de resina z350 xt, A2, esmalte, fotoactivadas con luz halógena (Bonart Plus), con un tiempo de exposición de 40 segundos, a una distancia de $0 \mathrm{~mm}$, fue de $2,26 \pm 03 \mathrm{~mm}$. Las muestras de resina $\mathrm{z} 350 \mathrm{xt}, \mathrm{A} 2$, esmalte, fotoactivadas con luz led de tercera generación (Radii Plus), con un tiempo de exposición de 10 segundos y una distancia $0 \mathrm{~mm}$, fue de 2,29 $\pm 03 \mathrm{~mm}$.

\section{Referencias}

[1] Rodríguez D, Pereira N. Evolución y tendencias actuales de las resinas. Acta Odontológica Venezolana [Internet]. 2008;46(3):1-19. Disponible en: http:// www.actaodontologica.com/ediciones/2008/3/evolucion_tendencias_resinas_compuestas.asp.

[2] Hervas A, Martínez MA, Cabanes J, Amaya E, Fos Galve P. Resinas compuestas. Revisión de los materiales e indicaciones clínicas. Med Oral Patol Oral Cir Bucal. 2006;11(2):215-20.

[3] Pires H. Comparação de dois sistemas de fotoativação de resinas compostas, led e lâmpada halógena: avaliação através de dureza em amostras de resina composta [tesis]. [Bauru]: Universidade de São Paulo; 2005. doi: http://dx.doi.org/10.11606/D.25.2005. tde-05092005-103811.

[4] Ilie N, Hickel R. Quality of curing in relation to hardness, degree of cure and polymerization depth measured on a nano-hybrid composite. Am J Dent [Internet]. 2007;20(4):263-8. Disponible en: http:// www.ncbi.nlm.nih.gov/pubmed/17907492.

[5] Carvalho Chain M, Baratieri LN. Restauraciones estéticas con resinas compuestas en dientes posteriores. Bogotá: Editorial Médica Panamericana; 2001.

[6] Krämer N, Lohbauer U, García-Godoy F, Frankenberger R. Light curing of resin-based composites in the LED era. Am J Dent [Internet]. 2008;21(3):13542. Disponible en: http://www.ncbi.nlm.nih.gov/ pubmed/18686762.

[7] Hiroyuki A, Takahito K, Koichi F, Hideo T, Seiji B. Effect of inhomogeneity of light from light curing units on the surface hardness of composite resin. Dent Mater J [Internet]. 2008;27(1):21-8. Disponible en: http://www.ncbi.nlm.nih.gov/pubmed/18309608.

[8] Yap A, Soh M. Curing efficacy of a new generation high-power LED lamp. Oper Dent [Internet]. 2005;30(6):758-63. Disponible en: http://www.ncbi. nlm.nih.gov/pubmed/16382599.

[9] Tarle Z, Knezevic A, Demoli N, Meniga A, Sutaloa J, Unterbrink G, et al. Comparison of composite curing parameters: Effects of light source and curing mode on conversion, temperature rise and polymerization shrinkage. Oper Dent. 2006;31(2):219-26. doi: http://dx.doi.org/10.2341/05-15. 
[10] Nomoto R, McCabe J, Hirano S. Comparison of halogen, plasma and LED curing units. Oper Dent. 2004;29(3):287-94.

[11] Sakaguchi RL, Douglas WH, Peters MC. Curing light performance and polymerization of composite restorative materials. J Dent. 1992;20(3):183-8.

[12] Veiga de Melo M, Ribeiro M, Rabelo J, Candido dos Reis A, Orbegoso V. Influencia de las unidades de curado led y luz halógena sobre la resistencia compresiva de las resinas compuestas. Acta Odontol Venez [Internet]. 2007 [citado 2016 jul 6]; 45(2):209-12. Disponible en: http:// www.scielo.org.ve/scielo.php?script=sci_arttext\&pid=S0001-63652007000200014\&lng=es.

[13] Rueggeberg FA. State-of-the-art: Dental photocuring - A review. Dent Mater. 2011;27(1):39-52.

[14] Santini A. Current status of visible light activation units and the curing of light-activated resin-based composite materials. Dent Update. 2010;37(4): 214-27.
[15] Kopperud H, Johnsen G, Lamolle S, Kleven IS, Wellendorf $\mathrm{H}$, Haugen HJ. Effect of short LED lamp exposure on wear resistance, residual monomer and degree of conversion for filtek z250 and tetricevoceram composites. Dent Mater. 2013;29(8):824-34. doi: http://dx.doi.org/10.1016/j.dental.2013.04.022.

[16] Caro M, Aizencop D, Ehrmantraut M. Profundidad de curado de resinas compuestas fluidas activadas con luz halógena y LED a través de distintos espesores de resina compuesta indirecta. Rev Dent Chile [Internet]. 2014 [citado 2015 jul 16]; 105(3):20-3. Disponible en: http://www.revistadentaldechile.cl/ temas $\% 20$ noviembre $\% 202014 /$ pdf/profundidad_ de_curado_web.pdf.

[17] Varanes Y, Autran F, Álvarez R, Gil-Mur F. Determinación de la profundidad de curado y propiedades mecánicas de composites dentales fotopolimerizables experimentales. RCOE [Internet]. 2005 [citado 2016 jul 7]; 10(2):161-70. Disponible en: http://scielo.isciii.es/scielo.php?script=sci_arttext\&pid=S1138-123X2005000200003.

[18] Shortal AC, Palin WM, Burtscher P. Refractive index mismatch and monomer reactivity. J Dent Res. 2008;87(2):84-8 